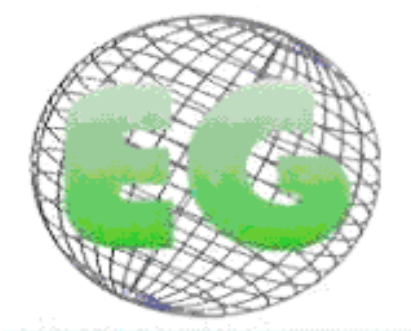

ISSN 1695-6141 N'25.

\title{
Intervenciones propuestas por las enfermeras hospitalarias para el seguimiento de pacientes con enfermedades crónicas
}

Interventions proposed by the hospital nurses for monitoring of patients with chronic diseases.

\author{
*Villarejo Aguilar, Luis \\ *Diplomado en Enfermería. Máster en Investigación e Innovación en Cuidados y Calidad de Vida. Distrito \\ Sanitario Jaén Norte. Atención Primaria. Servicio Andaluz de Salud.
}

Palabras clave: diagnósticos enfermeros, enfermedades crónicas, alta hospitalaria.

Keywords: nursing interventions; chronic diseases; discharge

\section{RESUMEN}

El presente estudio tiene como objetivo identificar las intervenciones enfermeras más frecuentes registradas en los Informes de Alta de pacientes con enfermedades crónicas. Se realizó un estudio descriptivo transversal sobre todos los pacientes dados de alta durante el período retrospectivo 2009-2010. La recogida de datos y análisis de las variables se hizo mediante revisión de los Informes de Alta. Como resultados principales se identificaron 59 intervenciones distintas y entre las más frecuentes estaban manejo de la energía, prevención de caídas, apoyo al cuidador familiar, prevención de las úlceras por presión, asesoramiento, apoyo emocional, ayuda en los autocuidados: AIVD, ayuda al auto cuidado y disminución de la ansiedad. La continuidad de los cuidados en los pacientes con enfermedades crónicas, dados de alta, resulta imprescindible de cara a orientar los cuidados intensivos proporcionados en el Hospital a unos cuidados crónicos para la prevención del deterioro provocado por las enfermedades crónicas.

\section{ABSTRACT}

This study aims to identify the most frequent nursing interventions recorded in the discharge reports for patients with chronic diseases. We conducted a cross sectional study on all patients discharged during the retrospective period 2009-2010. Data collection and analysis of variables was done by reviewing discharge reports. The main results identified 59 different interventions, of which themost common were power management, fall prevention family caregiver support, the prevention of pressure ulcers, advice, emotional support, Self-Care: IADL, self aid care and decreased anxiety. Continuity of care in discharged patients with chronic diseases is essential in orienting intensive care provided in the Hospital towards chronic care and so prevent deterioration caused by chronic illnessess. 


\section{INTRODUCCIÓN}

Las enfermedades crónicas representan uno de los principales retos a los que tienen que hacer frente los sistemas sanitarios; proyectados para dar respuesta a enfermedades y problemas de salud agudos.

La enfermedad crónica tiene una serie de características que necesita de un nuevo modelo de sistema de salud, ya que la prolongación en la esperanza de vida en ocasiones es a base de aumentar las plurimorbilidades, fundamentalmente crónicas.

Las enfermedades crónicas, por un lado, representan una importante limitación en la calidad de vida y estado funcional de las personas que la padecen y, por otro, son un generador del incremento de los costes sanitarios. Podría estimarse que el $75 \%$ del gasto sanitario en los países desarrollados y el $80 \%$ del gasto farmacéutico ${ }^{(1-4)}$

Por todo esto y para evitar que los pacientes con enfermedades crónicas entren en un mecanismo de feed-back negativo, es necesario identificar las intervenciones (5) que persiguen modificar el curso natural de las enfermedades del paciente, demorando su progresión y mejorando su nivel de salud y calidad de vida ${ }^{(6)}$

El potencial de la enfermería mediante la atención a la prevención y cuidados de las enfermedades crónicas parte desde una gestión y unos cuidados eficaces, desde las primeras fases, para permitir que los afectados por estas enfermedades crónicas lleven una vida plena y productiva ${ }^{(7)}$

La estandarización de los cuidados nos permitirá unificar criterios de actuación, sistematizar etapas y proporcionar un cuidado individualizado para cada paciente, con un criterio de actuación conjunto. Asimismo la aplicación del Proceso de Atención de Enfermería con taxonomías permite la sistematización de las etapas de los cuidados y sirve de guía para evitar la variabilidad de cada profesional ${ }^{(8)}$

Este estudio se plantea desde el convencimiento de que existen unas intervenciones más frecuentes propuestas por las enfermeras hospitalarias que ayuden a las enfermeras de atención primaria y sus cuidadoras a orientar los cuidados hacia la recuperación del estado funcional y la calidad de vida de los pacientes con enfermedades crónicas.

\section{MATERIAL Y MÉTODOS}

- DISEÑO: Descriptivo transversal.

- ÁMBITO Y SUJETOS DE ESTUDIO: El ámbito de estudio será un Hospital de tercer nivel y los sujetos de estudio serán todos los pacientes ingresados en el Hospital a los que se les haya hecho el Informe de Alta durante el período 2009-2010.

- RECOGIDA DE DATOS: Todos los datos serán recogidos de los Informes de Alta.

- ANÁLISIS DE DATOS: Análisis descriptivo univariante con frecuencias y porcentajes para variables categóricas y medias y desviación estándar para variables cuantitativas. 
- LIMITACIONES: La principal limitación del estudio es el sesgo de selección a la hora de seleccionar los pacientes con enfermedades crónicas a partir de la causa principal que originó su ingreso hospitalario y del proceso asistencial integrado. También el hecho de que la muestra sólo se ha tomado de un Hospital de referencia, pero de distintas Zonas Básicas lo que afectará a la validez externa del estudio.

\section{RESULTADOS}

Como resultado de los análisis de las intervenciones registradas en los Informes de Alta de los pacientes con enfermedades crónicas incluidos en la muestra se han determinado 59 intervenciones distintas, y se han formulado un total de 1010 intervenciones, lo que supone una mediana de 5 y un rango de 21. El número de intervenciones en cada plan de cuidados osciló entre 1 y 22.

Gráfico 1. Número de intervenciones registradas en los Informes de Alta de pacientes con enfermedades crónicas.

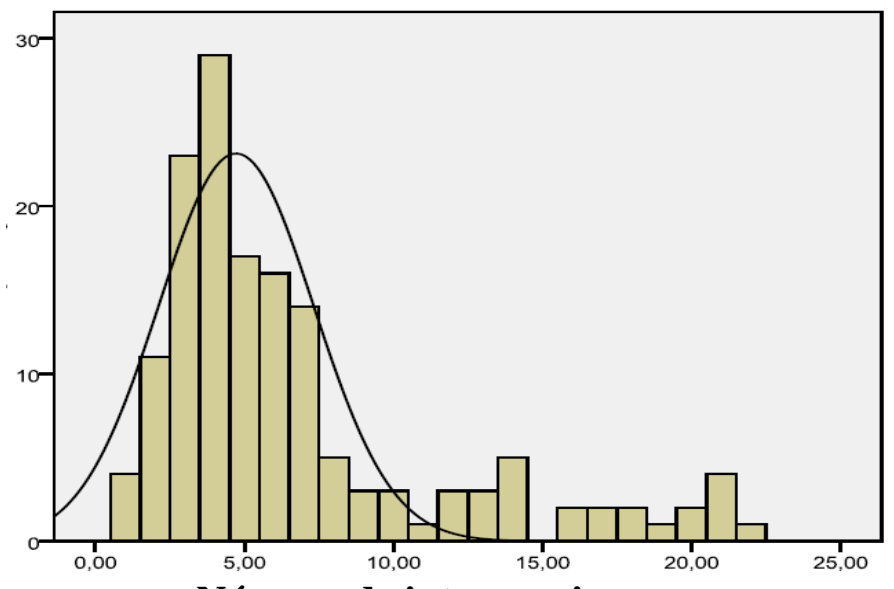

Número de intervenciones

Las intervenciones más frecuentes propuestas en los planes de cuidados al alta fueron: manejo de la energía $58,3 \%$, prevención de caídas $44,4 \%$, apoyo al cuidador familiar $42,4 \%$, prevención de las úlceras por presión $34,4 \%$, asesoramiento 30,5\%, apoyo emocional 29,8\%, ayuda en los autocuidados: AIVD 29,1\%, ayuda al auto cuidado 21,9\% y disminución de la ansiedad $21,2 \%$. 
Gráfico 2. Intervenciones presentes en los Informes de Alta de pacientes con enfermedades crónicas.

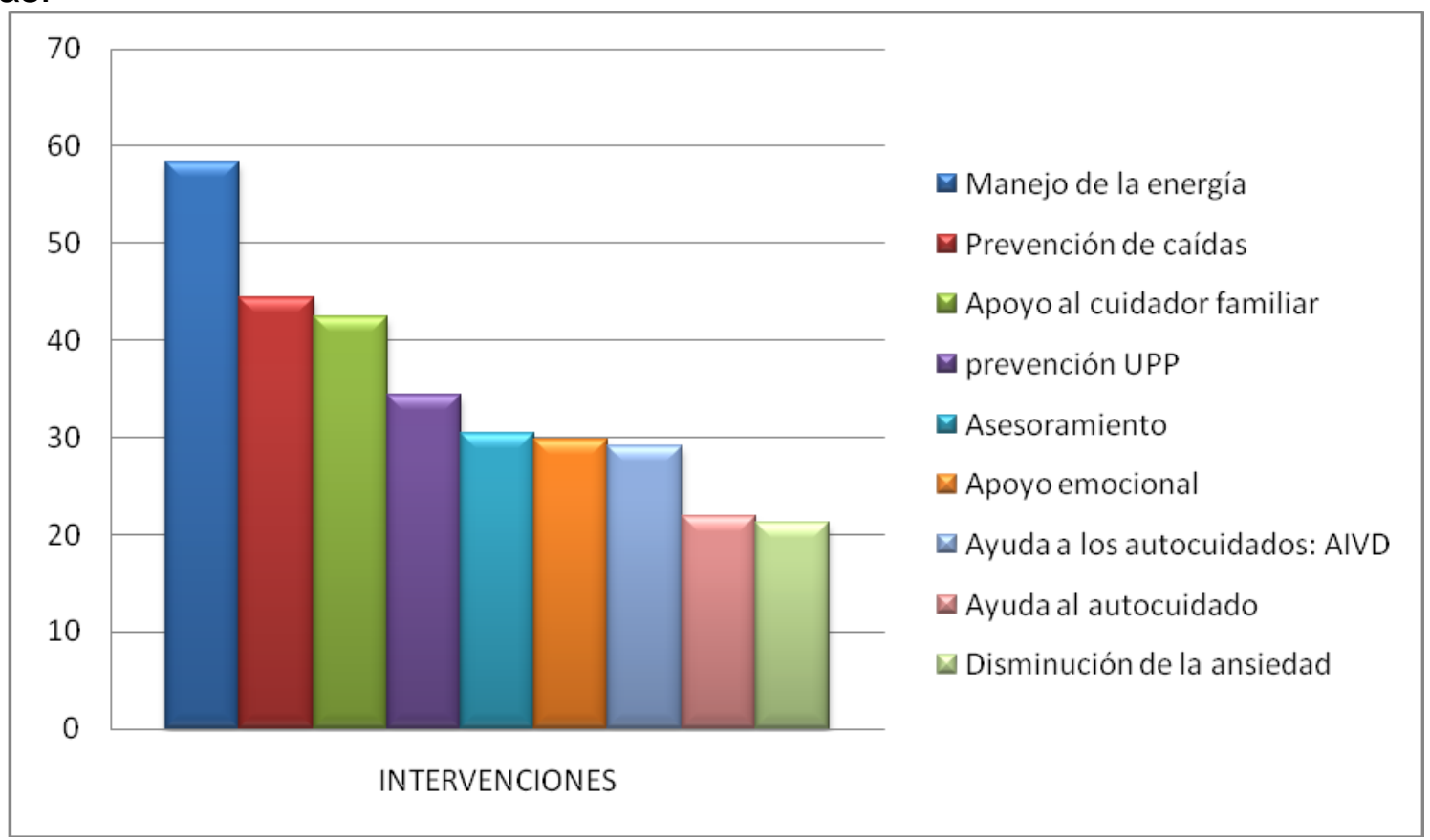

\section{DISCUSIÓN}

En la práctica de cada día, se cuida a los pacientes en base al juicio clínico de la enfermera que lo atiende y la asignación de unas u otras intervenciones no están influenciadas artificialmente.

En nuestros resultados se observa la concordancia entre la detección de problemas y los campos de intervenciones que se plantean para la solución de los problemas, en los que destacan como más frecuentes las agrupadas en los campos fisiológicos y conductuales.

Destacan como puntos fuertes en las intervenciones la ayuda a los autocuidados, las relacionadas con la enseñanza del paciente, la prevención y promoción de la salud, y la disminución del dolor y la ansiedad, estos datos coinciden con los aportados por García Fernández FP et al. en pacientes pluripatológicos ${ }^{(6)}$ y difieren de los encontrados por Almeida et al. ${ }^{(9)}$

Otras intervenciones frecuentes propuestas por las enfermeras hospitalarias al alta son las relacionadas con la prevención de riesgos como prevención de caídas o prevención de úlceras por presión, derivadas del alto índice de edad y la limitación funcional que presentan la mayoría de éstos pacientes. También aparecen entre las más frecuentes intervenciones relacionadas con las cuidadoras como apoyo emocional y asesoramiento quizás para promover el seguimiento por parte de las enfermeras de atención primaria y sigan contribuyendo a aliviar la carga que supone el rol de cuidadora.

Como conclusiones señalar que se deben orientar los cuidados hacia intervenciones efectivas y eficaces desde las primeras fases para prevenir el deterioro progresivo provocado por las enfermedades crónicas. 


\section{BIBLIOGRAFÍA}

(1) De Sancho Martín JL, Sarria Santamera A, Yáñez Cadena D, Hernández Torres A, Ovalle Perandones MA, Perianes Rodríguez A. Revisión de intervenciones en atención primaria para mejorar el control de las enfermedades crónicas. Agencia de Evaluación de Tecnologías Sanitarias, Instituto de Salud Carlos III. Ministerio de Sanidad y Consumo. Madrid; 2003.

(2) Sociedad Española de Geriatría y Gerontología. Con motivo del Día Europeo e Internacional de la Discapacidad. Madrid, 2 de diciembre de 2005.

(3) El sistema sanitario y la atención a las personas en situación de dependencia en España. Libro blanco. Ministerio de Trabajo y Asuntos Sociales. Secretaría de Estado de Servicios Sociales, familias y discapacidad. IMSERSO; 2004.

(4) Casado Martín D. Los efectos del envejecimiento demográfico sobre el gasto sanitario: mitos y realidades. Gac Sanit 2000; 15(2): 154-163.

(5) McCloskey J C, Bulecheck G M. Clasificación de Intervenciones de Enfermería. Nursing Interventions Clasification (3 $3^{\underline{a}}$ ed.). Madrid: Harcourt, 2002.

(6) García Fernández FP, Carrascosa García MI. Resultados de las intervenciones enfermeras en el proceso pluripatológico para mejorar la práctica clínica. Gerokomos 2008; 19(4): 170-176.

(7) Consejo Internacional de Enfermeras.CIE. Con motivo del Día Internacional de la Enfermería. Madrid, 12 de mayo 2010.

(8) Alfaro,R. Aplicación del proceso de enfermería (2 ed.). Barcelona: Doyma Enfermería, 1992.

(9) Almeida MA, Aliti GB, Franzen E, Thomé EGR, Unicovsky MR, Rabelo ER, Ludwig MLM, Moraes MA. Diagnósticos de enfermería e intervenciones prevalentes en el cuidado del anciano hospitalizado. Rev Latino-am Enfermagem 2008 julo-agosto ;16(4).

ISSN 1695-6141

๑ COPYRIGHT Servicio de Publicaciones - Universidad de Murcia 